\title{
Fine-needle-aspiration (FNA) Cytology for SRC Type Breast Cancer: 13 Case Reports \\ C Zhao ${ }^{1}$, X Wang ${ }^{2}$, LWang ${ }^{3}$
}

\begin{abstract}
Objective: To investigate the cytohistological findings of 13 breast carcinoma cases with SRCs

Methods: From January 2006 to August 2015, 13 cases of SRC type breast cancer had undergone FNA cytology examination before excisional biopsy or operation. All the histological sections of each case were confirmed to have at least $20 \%$ SRCs in the tumor cells. The clinicopathological features of SRC type breast cancer were investigated.

Results: Of the 13 breast carcinoma cases, there were invasive ductal carcinoma (IDC) ( $n=5)$, invasive lobular carcinoma (ILC) (n=5), pleomorphic lobular carcinoma (PLC) $(n=1)$, and mucinous carcinoma (MC) (n=2). 3 cases of IDC, 1 cases of PLC, and 1 case of MC had grade 2 or 3 nuclear atypia, whereas 1 case of IDC, 4 cases of ILC, and 1 case of MC had grade 1 nuclear atypia. Axillary lymph nodes were positive for metastatic carcinoma in 1 case with IDC and 1 case with ILC. In fine needle aspiration smears, the intracytoplasmic lumen (ICL) type SRCs were found to be major constituents of SRCs in ILC and PLC, while non-ICL type SRCs were predominant in IDC. MC tumors had SRCs of both ICL and non- ICL types. During our follow-up period ranging from 97 to $3650(1458 \pm 1055)$ days, all the patients were alive without recurrence of the tumor.
\end{abstract}

Conclusion: Our results indicate that the nature of SRCs may vary with the histological subtype of breast cancer, but its presence may not indicate poor prognosis.

Keywords: Breast cancer, cytology, fine needle aspiration, signet ring cells

From: ${ }^{1}$ Department of Ultrasound Diagnosis, and ${ }^{2}$ Department of Breast Surgery, The Affiliated Hospital of Qingdao University, Qingdao, ${ }^{3}$ Department of Ultrasound Diagnosis, The Affiliated Hospital of Shandong University, Jinan, China.

Correspondence: Professor C Zhao, Department of Ultrasound Diagnosis, The Affiliated Hospital of Qingdao University, No 16 Jiangsu Road, Qingdao, China. Fax: 86-532-82911834, e-mail:kqfjncs@126.com 


\section{INTRODUCTION}

Signet ring cell (SRC) carcinoma is a unique subtype of mucin-producing adenocarcinoma arising in various organs such as the stomach, colon, lungs, and prostate gland (1-4). The presence of SRCs in breast cancer was first reported by Saphir more than half a century ago (5). Steinbrecher et al. described 5 cases of SRC type of breast cancer as an uncommon type of breast cancer characterized by the presence of intracellular mucin cells without large amounts of extra-cellular mucin (6). Hull et al. later found that SRC type of breast cancer were extremely rare, which were only comprised about $4.5 \%$ of all the breast cancers (7-9). In 2012, World Health Organization has included a section on 'carcinomas with SRC differentiation' in the chapter on mucinous carcinoma (MC); however, it remains undetermined whether breast cancer with SRCs should represent a distinct disease entity (10).

Histological characteristics of breast carcinoma with SRCs have been elusive because of the conflicting results from earlier studies. Some studies have demonstrated that breast cancers with SRCs are generally invasive lobular carcinomas (ILC) (11-12). However, Kelten et al. has reported 10 cases of SRCs type of breast cancer, about which 7 cases were invasive ductal carcinoma (IDC), 2 cases were ILC, and the remaining was a mixture of IDC and ILC (13). Other studies have found that the presence of SRCs has the similar frequency in IDC and ILC (14-15). Regardless of the origin, breast cancer with SRCs behaves more aggressively than other breast cancers without SRCs $(7,11-12)$. Therefore, it is crucial to identify the presence of SRCs during histological diagnosis of breast cancers. Accordingly, it is important to recognize SRCs in fine needle aspiration (FNA) cytology smears as well, which is usually performed prior to surgical resection of the breast tumor.

In the present study, we investigated the clinicopathological features of breast carcinomas 
with SRCs. The SRCs were classified into intracytoplasmic lumen (ICL) type and non-ICL type according to the definition described previously $(10,16)$. We also retrospectively reviewed FNA findings of each case to describe cytomorpholoigcal characteristics of breast carcinoma with SRCs.

\section{SUBJECTS AND METHODS}

\section{Case selection}

We searched the archives at the Department of Diagnostic Pathology, Shandong University (China) between January 2006 and August 2015. We found 29 cases with "SRC" in the diagnostic lines and/or histological description. Patients with breast cancer metastasized from primary carcinomas to the distant organs were excluded. We chose 13 cases that had undergone FNA cytology examination before excisional biopsy or operation. We reviewed all the histological sections of each case to confirm that at least $20 \%$ of the tumor cells consisted of SRCs,based on a previous report that stated that the presence of SRCs accounting for more than $20 \%$ of the tumor should have clinical significance (11).

FNA cytology was performed by a number of experienced surgeons by using a 20-gauge needle attached to a $20 \mathrm{~mL}$ syringe. Clinical data were extracted and reviewed from medical charts and pathology reports. This study was conducted in accordance with the principles embodied in the Declaration of Helsinki and informed consent was obtained from each patient for the use of their tissues samples.

\section{Special and immunohistochemical stainings}

Specimens were smeared on glass slides and immediately fixed with solution containing 95\% 
ethanol. Subsequently, the cytological slides were stained according to the routine Papanicolaou method. Formalin-fixed histological sections of the tumors were stained with alcian blue $(\mathrm{AB})(\mathrm{pH}$ 2.5) and periodic acid-Schiff (PAS) to reveal both acidic (blue) and neutral (red) mucins (17). Immunohistochemical staining was performed with primary antibodies by using the standard avidin-biotin-peroxidase complex technique. The following antibodies were used: E-cadherin (NCH-38, dilution 1:50; DAKO, Denmark), GCD-FP-15 (NCL-GCDFP15, dilution 1:20; Novocast-ra Laboratories, UK), monoclonal rabbit anti-human estrogen receptor (ER) (SP1, dilution 1:1; Ventana Medical Systems, USA), monoclonal rabbit anti-human progesterone receptor $(\mathrm{PgR})(1 \mathrm{E} 2$, dilution 1:1; Ventana Medical Sy-stems), monoclonal rabbit anti-human HER2/ neu (4B5, dilution 1:1; Ventana Medical Sys-tems), monoclonal mouse anti-human Ki67 (M7240, dilution 1:100; DAKO, Denmark), polyclonal rabbit anti-human chromogranin A (A0430, dilution 1:100; DAKO, Denmark) and monoclonal mouse anti-human synaptophysin (NCLSYNAP-299, dilution 1:100; Novocastra, UK). Positive and negative controls for each antibody were used. Positivity for ER and PgR was graded according to the guidelines established at the St Gallen Consensus Conference (18).

Briefly, ER and PgR status should be judged as positive (+) if any positive cells are detected within the tumor. Assessment of HER2 follows the guidelines defined by American Society of Clinical Oncology/College of American Pathologists (19). Positivity for PAS, AB, and other immunohistochemical staining was expressed as negative (-), mild (+), moderate $(++)$, or strong (+++).

\section{Review of FNA cytology smears}


We examined FNA smears to assess the morphological features of SRCs. An SRC was defined as a cell with a single large vacuole within the cytoplasm. We further classified SRCs into ICL type and non-ICL type based on the definition described previously $(10,16)$. Specifically, ICL type SRCs are characterized by a "target" appearance owing to the presence of a large ICL containing PAS or AB positive globules. Non-ICL type SRCs contain acidic muco-substance that diffusely fills the cytoplasm and dislodges the nucleus to one pole of the cell, as seen in gastric carcinoma.

The presence of ICL or non-ICL type SRCs was recorded as present (+) or absent (-). Cells with multiple vacuoles or bubbles were excluded since degenerative cells can also resemble SRCs. The smears were also evaluated for the following cytomorphological features: cellularitylow $(+)$, moderate $(++)$, or high $(+++)$; nuclear size-small $(+)$, moderate $(++)$, or large $(+++)$; nuclear atypia-mild $(+)$, moderate $(++)$, or severe $(+++)$; chromatin-fine $(+)$ or coarse $(++)$; ncleolus-distinct $(+)$ or indistinct $(-)$; nuclear pleomorphism-mild $(+)$, moderate $(++)$, or severe $(+++)$; background necrosis-present (+) or absent (-); and discohesiveness-mild (+), moderate (++), or severe $(+++)$. The size of nucleus was determined in comparison with that of red blood cells (RBC) as mild: less than $2 \times \mathrm{RBC}$, moderate: $2-3 \times \mathrm{RBC}$, and large: greater than $3 \times \mathrm{RBC}$ as described elsewhere (20). A review of the cytological diagnosis and cytohistological spe-cimens was performed by two pathologists (R.O and T.S).

\section{RESULTS}

\section{Clinicopathological profiles of breast carcinoma with SRCs}

Table 1 summarizes patient and tumor characteristics of 13 patients of SRC type of breast cancer. The diagnosis of lobular carcinoma was confirmed by detecting the loss of E-cadherin expression. 
Histological variants of the tumors consisted of IDC of no special type $(n=5)$, ILC $(n=5)$, pleomorphic lobular carcinoma (PLC) $(\mathrm{n}=1)$, and MC (n=2) (Figure 1A-D). The patients' age ranged from 39 to 85 (mean $60.2 \pm 13$ ) years. The tumor size ranged from 0.7 to 3.5 (mean $2.0 \pm$ 0.8) $\mathrm{cm}$. Three cases of IDC, 1 case of PLC and 1 case of MC had grade 2 or 3 nuclear atypia, whereas 1 case of IDC, 4 case of ILC, and 1 case of MC had grade 1 nuclear atypia. Lymphovascular invasion was observed in 2 cases with IDC, 5 cases with ILC, and in none with PLC and MC. Axillary lymph nodes were positive for metastatic carcinoma in 1 case with IDC and in 1 case with ILC. None exhibited distant metastasis. During the clinical follow-up period that ranged from 97 to 3650 (mean $1458 \pm 1055$ ) days, all the patients stayed alive, with no recurrence of the tumors

\section{AB, PAS, and immunohistochemical staining}

Table 2 summarizes special staining and immunohistochemical profiles of 11 patients with SRC type breast cancer. As for the hormonal status of the tumor cells, 1 case with IDC, 1 case with PLC and 1 case with MC showed a triple negative phenotype, 1 case with IDC showed HER2 type, and all the others showed type A. SRCs of all the cases showed positive PAS staining with predominance in IDC cases (Figure 1E). By contrast, $\mathrm{AB}$ staining was more conspicuous in SRCs of ILC compared with those of IDC (Figure 1F). SRCs of PLC and MC displayed similar staining intensity for PAS and AB. GCDFP-15 expression was focally or diffusely identified in 3 cases with IDC, 3 cases with ILC, 1 cases with PLC, and in 1 cases with MC. Chromogranin A was focally positive in SRCs of 1 case with MC, but none showed significant expression of synaptophysin.

\section{Review of FNA cytology reports and detailed characteristics of FNA smears}

Table 3 summarizes cytological findings of the FNA smears. ICL type SRCs were seen in all the ILC and PLC cases, and in 2 of the 5 IDC cases (Figure 2A-E). Non-ICL type SRCs were present 
in 3 IDC cases, but were identified in only 1 ILC case, and were absent in the PLC case (Figure 2B, 2F). MC cases had both ICL and non-ICL type SRCs. These findings suggest that ICL type SRCs are more commonly seen in ILC than in IDC, consistent with the findings of a previous report (16). All the patients showed moderate or high degree of cellularity, except for one patient with IDC who showed low cellularity.

Nuclear size was moderate in all IDC cases, large in all MC and PLC cases, and mild to moderate in ILC cases. Nuclear atypia was severe in 1 IDC and 1 PLC cases, moderate in 3 IDC and 2 MC cases, and mild in all ILC cases. Fine chromatin was seen in all ILC cases, while coarse chromatin was predominant in the other cases. Nucleolus was distinct in 3 ILC cases and 1 PLC case, and indistinct in the other cases. Pleomorphism was severe in the PLC case, and moderate in 1 IDC case and $1 \mathrm{MC}$ case, and mild in all the other cases. Necrosis was identified only in the background of PLC case. Discohesiveness was severe in all MC cases and 2 ILC cases, and either mild or moderate in the other cases.

\section{DISCUSSION}

SRCs are one of the morphological findings commonly encountered in the aspirates of the breast tumors, defined as cells containing intracytoplasmic vacuoles inducing peripheral displacement and indentation of nuclei. Other studies revealed that SRCs contain secretory products, such as mucin, as seen in adenocarcinoma in other organs (21). Despite the recognition of SRCs in breast tumors, the origin of SRCs has been a matter of debate over the last few decades. Previous studies have found that a vast majority of SRCs in the breast cancer originate from ILC (11-12). Contrary to these reports, other studies have revealed that the presence of SRCs has been noted with similar 
frequency in IDC and ILC in the histological specimens, suggesting that the presence of SRCs does not necessarily predominate in $\operatorname{ILC}(14,15)$. We assume that the above-mentioned differences in the presence of SRCs may be due to the different definition of SRCs used by each study. To delineate the cytological features of SRCs, Kamiya et al subgrouped SRCs into ICL type and nonICL type cells (16). ICL type cells had large ICL with a varying amount of mucin, while non-ICL cells had wide amorphous cytoplasm diffusely dispersed with mucin. In the present study, we chose SRCs based on the criteria put forth by Kamiya et al and found 4 cases of IDC and 4 cases of ILC, suggestive of no significant difference in the frequency of SRC presence in IDC and ILC. However, given that IDC and ILC account for $80 \%$ and $15 \%$, respectively, of the breast carcinomas treated at our institution, it is difficult to estimate the percentage of SRCs contained in ILC and IDC.

A notable finding in our study is the case of PLC with SRCs. The PLC was first described by Dixon et al (22). It exhibits the infiltrating patterns of classic ILC with pleomorphic nuclei and often shows aggregation of tumor cells. Compared with the FNA smears of conventional ILC, those of PLC are more cellular, with 2 or 3 times larger nuclei, showing irregular contours, prominent nucleoli, hyperchromasia, and high mitotic activity (23-25). These cytological features often overlap with those of IDC, resulting in erroneous diagnoses (26). Clinically, patients with PLC tend to have a poor prognosis, explained by the advanced stage at presentation, and they are more likely to develop distant metastasis and recurrence than those with the classical form of ILC (2728). In our case of PLC, irregular contours, prominent nucleoli, and hyperchromasia were noted, as reported previously. In addition to these features, our PLC case presented with SRCs. To our knowledge, there are only a few reports of the presence of SRCs in PLC; thus, the presence of SRCs in PLC remains under-recognized (29-30). Our study indicates that PLC should be included in the differential diagnoses when SRCs are identified in FNA smears. 
Regardless of the primary organs affected, carcinomas with SRCs tend to have an aggressive clinical course due to frequent metastasis to lymph nodes (1-4). However, the prognosis of breast carcinoma with SRCs varies depending on the study, probably because the diagnostic criteria for SRC carcinoma have not been strictly defined. Merino et al demonstrated that ILC in which SRCs account for more than $20 \%$ of the tumor cells showed a poor prognosis: $60 \%$ of the patients died within 7 years, and some of them exhibited unusual metastatic pattern, with a propensity to involve serosal surfaces of gastrointestinal tracts (11).

Frost et al showed that the presence of $10 \%$ or more SRCs represents a poor individual prognostic factor in patients with ILC due to frequent recurrence or distant metastasis (21). In our study, only 2 of the 11 patients evaluated showed lymph node metastasis even though SRCs accounted for more than $20 \%$ of tumor cells in all the patients. Based on our results, we are unable to conclude that the presence of SRCs could be an adverse prognostic factor for breast cancer. A study evaluating a higher number of SRC cases, with a longer follow-up period, is needed to determine the clinical significance of SRCs in breast cancer.

In our study, 2 cases exhibited histological features of MC. One may argue that these cases should not be considered as carcinoma with SRCs, as some studies have suggested that carcinoma with SRCs should be distinguished from MC $(6,31)$. In contrast, Kuroda et al propose that there should be a distinct type of MC characterized by the presence of SRCs, apart from MC lacking the presence of SRCs (32). Although it remains controversial how these cases should be categorized, further studies using a larger number of similar cases with correlations to clinical profiles are needed to clarify the clinical significance of SRCs in MC. 


\section{CONCLUSION}

We examined the clinicopathological features of 13 cases with breast carcinoma containing SRCs, consisting of 5 cases of IDC, 5 of ILC, 1 of PLC and 2 of MC. In FNA smears, while ICL type SRCs were predominant in ILC, non-ICL type SRCs were conspicuous in IDC. MC showed SRCs of both ICL and non-ICL types. During our follow-up period, no patient exhibited clinical signs of recurrence. We assume that the nature of the SRCs may differ depending on the histological subtype of breast cancer; however, their clinical significance remained undetermined.

\section{ACKNOWLEDGEMENTS}

This study was supported by a grant from Shandong Natural Scientific Research Fond (N0:2014ZM4672) and National Natural Scientific Research Fond (No: 76530284).

\section{AUTHORS' NOTE}

Cheng Zhao conceived paper, oversaw data collection, conducted data analysis, wrote manuscript and revision of manuscript and approved final version. Cheng Zhao, Xingang Wang and Lihua Wang participated in study design and approved final version. The authors declare that they have no conflicts of interest. 


\section{REFERENCES}

1. Boland JM, Wampfler JA, Jang JS, Wang X, Erickson-Johnson MR, Oliveira AM, et al. Pulmonary adenocarcinoma with signet ring cell features: a comprehensive study from 3 distinct patient cohorts. Am J Surg Pathol 2014; 38: 1681-8.

2. Hugen N, van de Velde CJ, de Wilt JH, Nagtegaal ID. Metastatic pattern in colorectal cancer is strongly influenced by histological subtype. Ann Oncol 2014; 25: 651-7. 
3. Huh CW, Jung dH, Kim JH, Lee YC, Kim H, Yoon SO, Yet al. Signet ring cell mixed histology may show more aggressive behavior than other histologies in early gastric cancer. J Surg Oncol 2013; 107: 124-9.

4. Humphrey PA. Histological variants of prostatic carcinoma and their significance. Histopathology 2012; 60: 59-74.

5. Saphir O. Signet-ring cell carcinoma. Mil Surg 1951; 109: 360-9.

6. Steinbrecher JS, Silverberg SG. Signet-ring cell carcinoma of the breast. The mucinous variant of infiltrating lobular carcinoma? Cancer 1976; 37: 828-40.

7. Hull MT, Seo IS, Battersby JS, Csicsko JF. Signet-ring cell carcinoma of the breast: a clinicopathologic study of 24 cases. Am J Clin Pathol 1980; 73: 31-5.

8. Li X, Feng YF, Wei WD, Liu P, Wang J, Xie XM. Signet-ring cell carcinoma of the breast: a case report. World J Surg Oncol 2013; 11: 183.

9. Karabagli P, Kilic H. Primary pure signet cell carcinoma of the breast: a case report and review of the literature. Breast Cancer 2016; 20: 363-6.

10. Bussolati G, Sapino A. WHO Classification of Tumours of the Breast, 4th edition. In: Lakhani SR, Ellis IO, Schnitt SJ, Tan PH, van de Vijver MJ, editors. Lyon, France: IARC Press; 2012 pp 60-1.

11. Merino MJ, Livolsi VA. Signet ring carcinoma of the female breast: a clinicopathologic analysis of 24 cases. Cancer 1981; 48: 1830-7.

12. Raju U, Ma CK, Shaw A. Signet ring variant of lobular carcinoma of the breast: a clinicopathologic and immunohistochemical study. Mod Pathol 1993; 6: 516-0.

13. Kelten C, Akbulut M, Zekioglu O, Kapkaç M, Erhan Y, Ozdemir N. Signet ring cells in fine needle aspiration cytology of breast carcinomas: review of the cytological findings 
in ten cases identified by histology. Cytopathology 2009; 20: 321-7.

14. Sethi S, Cajulis RS, Gokaslan ST, Frias-Hidvegi D, Yu GH. Diagnostic significance of signet ring cells in fine-needle aspirates of the breast. Diagn Cytopathol 1997; 16: $117-$ 21.

15. Sturgis CD, Sethi S, Cajulis RS, Hidvegi DF, Yu GH. Diagnostic significance of 'benign pairs' and signet ring cells in fine needle aspirates (FNAs) of the breast. Cytopathology 1998; 9: 308-19.

16. Kamiya M, Mizuguchi K, Yoshimoto M, Tanaka M, Motegi S, Matushima H, Ishizawa M, Nakamura K. Cytologic diagnosis of signet-ring cell carcinoma of the breast. Acta Cytol 1998; 42: 650-6.

17. Sáez C, Japón MA, Poveda MA, Segura DI. Mucinous (colloid) adenocarcinomas secrete distinct O-acylated forms of sialomucins: a histochemical study of gastric, colorectal and bre-ast adenocarcinomas. Histopathology 2011; 39: 554-60.

18. Goldhirsch A, Ingle JN, Gelber RD, Coates AS, Thürlimann B, Senn HJ; Panel members. Thresholds for therapies: highlights of the St Gallen International Expert Consensus on the primary therapy of early breast cancer 2009. Ann Oncol 2009; 20: 1319-29.

19. Wolff AC, Hammond ME, Hicks DG, Dowsett M, McShane LM, Allison KH et al. Recommendations for human epidermal growth factor receptor 2 testing in breast cancer: American Society of Clinical Oncology/College of American Pathologists clinical practice guideline update. Arch Pathol Lab Med 2014; 138: 241-56.

20. Cyrta J, Andreiuolo F, Azoulay S, Balleyguier C, Bourgier C, Mazouni C et al. Pure and mixed mucinous carcinoma of the breast: fine needle aspiration cytology findings and review of the literature. Cytopathology 2013; 24: 377-84. 
21. Frost AR, Terahata S, Yeh IT, Siegel RS, Overmoyer B, Silverberg SG. The significance of signet ring cells in infiltrating lobular carcinoma of the breast. Arch Pathol Lab Med 1995; 119: 64-8.

22. Dixon JM, Anderson TJ, Page DL, Lee D, Duffy SW. Infiltrating lobular carcinoma of the breast. Histopathology 1982; 6: 149-61.

23. Weidner N, Semple JP. Pleomorphic variant of invasive lobular carcinoma of the breast. Hum Pathol 1992; 23: 1167-71.

24. Dabbs DJ, Grenko RT, Silverman JF. Fine needle aspiration cytology of pleomorphic lobular carcinoma of the breast. Duct carcinoma as a diagnostic pitfall. Acta Cytol 1994; 38: $923-6$.

25. Auger M, Hüttner I. Fine-needle aspiration cytology of pleomorphic lobular carcinoma of the breast. Comparison with the classic type. Cancer 1997; 81: 29-32.

26. Gangane N, Anshu, Shivkumar VB, Sharma S. Pleomorphic lobular carcinoma of the breast. A case report. Acta Cytol 2002; 46: 909-11.

27. Bentz JS, Yassa N, Clayton F. Pleomorphic lobular carcinoma of the breast: clinicopathologic features of 12 cases. Mod Pathol 1998; 11: 814-22.

28. Butler D, Rosa M. Pleomorphic lobular carcinoma of the breast: a morphologically and clinically distinct variant of lobular carcinoma. Arch Pathol Lab Med 2013; 137: 168892.

29. Fadare O. Pleomorphic lobular carcinoma in situ of the breast composed almost entirely of signet ring cells. Pathol Int 2016; 56: 683-7.

30. Miller MJ, Cazzaniga M, Casadio C. Cytologic findings of breast ductal lavage and concurrent fine needle aspiration in pleomorphic lobular carcinoma in situ: a case report. 
Acta Cytol 2008; 52: 207-210.

31. Al-Hariri JA. Primary signet ring cell carcinoma of the breast. Virchows Arch A Pathol Anat Histol 1980; 388: 105-11.

32. Kuroda N, Fujishima N, Ohara M, Hirouchi T, Mizuno K, Lee GH. Invasive ductal carcinoma of the breast with signet-ring cell and mucinous carcinoma components: diagnostic utility of immunocytochemistry of signet-ring cells in aspiration cytology materials. Diagn Cytopathol 2007; 35: 171-1. 
Table 1: Clinicopathological profiles of 13 patients with breast carcinoma with signet ring cells

\begin{tabular}{llllllllllll}
\hline case & Age(years) & DX & Side & Size(cm) & $\begin{array}{l}\text { SR } \\
\text { C(\%) }\end{array}$ & NG & Ly & V & N & M & FU(days) \\
\hline 1 & 48 & IDC & R & 2.6 & 20 & 2 & - & - & - & - & 102 \\
2 & 57 & IDC & R & 1.8 & 80 & 3 & + & + & - & - & 3653 \\
3 & 72 & IDC & R & 1.4 & 20 & 2 & - & - & + & - & 1982 \\
4 & 34 & IDC & R & 0.7 & 20 & 1 & - & - & - & - & 893 \\
5 & 46 & IDC & L & 1.6 & 40 & 1 & - & - & - & - & 1624 \\
6 & 29 & ILC & L & 3.5 & 60 & 1 & + & - & - & - & 2137 \\
7 & 78 & ILC & R & 4.1 & 30 & 1 & + & + & - & - & 1782 \\
8 & 63 & ILC & L & 1.2 & 20 & 1 & + & - & + & - & 354 \\
9 & 66 & ILC & R & 2.4 & 40 & 1 & + & - & - & - & 2137 \\
10 & 41 & ILC & R & 1.8 & 60 & 1 & + & + & - & - & 398 \\
11 & 43 & PLC & R & 0.8 & 20 & 3 & - & - & - & - & 413 \\
12 & 59 & MC & R & 1.8 & 40 & 2 & - & - & - & - & 432 \\
13 & 36 & MC & R & 2.3 & 40 & 1 & - & - & - & - & 2254 \\
\hline
\end{tabular}

Abbreviations: Dx, histological diagnosis; SRC, signet ring cell; NG, nuclear grade; ly, lymphatic invasion; $\mathrm{v}$, venous invasion; $\mathrm{N}$, lymph node metastasis; $\mathrm{M}$, distant metastasis; FU, follow-up period; IDC, invasive ductal carcinoma, IDC, invasive lobular carcinoma; PLC, pleomorphic lobular carcinoma; MC, mucinous carcinoma

Table 2: AB and PAS Stainings, and Immunohistochemical Profiles

\begin{tabular}{llllllllll}
\hline Case & ER & PgR & HER2 & $\begin{array}{l}\text { Ki67 } \\
(\%)\end{array}$ & PAS & AB & GCDFP15 & CGA & SYN \\
\hline 1 & - & - & - & 5 & +++ & ++ & ++ & - & - \\
2 & + & + & - & 18 & ++ & + & + & - & - \\
3 & - & - & + & 23 & +++ & + & +++ & - & - \\
4 & + & - & - & 7 & + & ++ & - & - & - \\
5 & + & + & - & 5 & + & ++ & - & - & - \\
6 & + & + & - & 20 & + & +++ & + & - & - \\
7 & + & - & - & 15 & + & ++ & +++ & - & - \\
8 & + & + & - & 15 & + & ++ & + & - & - \\
9 & + & - & - & 12 & + & + & +++ & - & - \\
10 & - & - & - & 5 & ++ & ++ & + & - & - \\
11 & + & + & - & 5 & + & + & + & - & - \\
12 & + & - & - & 15 & +++ & +++ & - & ++ & - \\
13 & - & - & - & 20 & +++ & ++ & ++ & - & - \\
\hline
\end{tabular}

Abbreviations: ER, estrogen receptor; PgR, progesterone receptor; PAS, Periodic acid-Schiff; $\mathrm{AB}$, alcian blue; GCDFP, gross cystic disease fluid protein; CGA, chromogranin A; SYN, synaptophysin. 
Table 3: Cytomorphology of fine needle aspiration cytological samples

\begin{tabular}{|c|c|c|c|c|c|c|c|c|c|c|}
\hline \multirow[b]{2}{*}{ Case } & \multicolumn{2}{|c|}{ SRC type } & \multirow[b]{2}{*}{ Cellularity } & \multirow{2}{*}{$\begin{array}{l}\text { Nuclear } \\
\text { size }\end{array}$} & \multirow[b]{2}{*}{ Atypia } & \multirow[b]{2}{*}{ Chromatin } & \multirow[b]{2}{*}{ Nucleous } & \multirow[b]{2}{*}{ Pleomorphism } & \multirow[b]{2}{*}{ Necrosis } & \multirow[b]{2}{*}{ Discohesiveness } \\
\hline & ICL & Non-ICL & & & & & & & & \\
\hline 1 & - & - & ++ & ++ & +++ & ++ & - & + & - & ++ \\
\hline 2 & + & + & ++ & ++ & ++ & + & + & + & - & + \\
\hline 3 & - & + & + & + & ++ & ++ & - & ++ & - & + \\
\hline 4 & - & - & ++ & ++ & + & + & - & + & - & + \\
\hline 5 & + & - & +++ & ++ & + & + & - & + & - & ++ \\
\hline 6 & + & - & +++ & +++ & ++ & +++ & + & + & - & + \\
\hline 7 & + & + & +++ & +++ & + & + & + & + & - & +++ \\
\hline 8 & + & - & +++ & ++ & + & + & + & + & - & ++ \\
\hline 9 & + & - & + & ++ & + & + & + & + & + & ++ \\
\hline 10 & + & - & +++ & + & + & ++ & - & + & - & +++ \\
\hline 11 & + & + & ++ & ++ & + & + & - & + & - & +++ \\
\hline 12 & + & + & ++ & ++ & ++ & ++ & - & +++ & - & +++ \\
\hline 13 & + & + & +++ & +++ & ++ & +++ & - & + & - & +++ \\
\hline
\end{tabular}



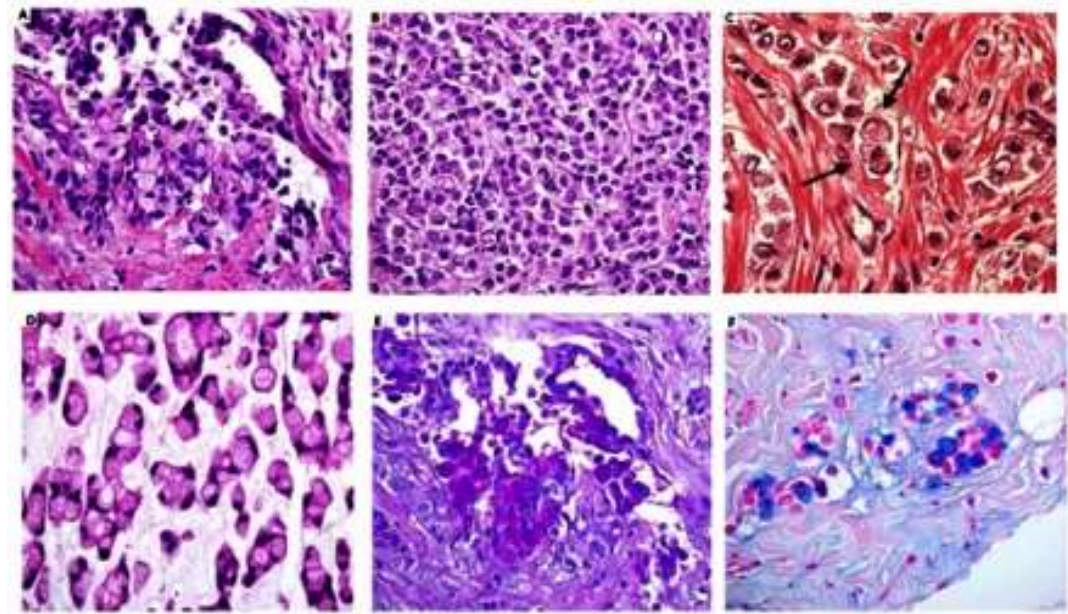

Fig. 1: Signet ring cells (SRCs) of invasive ductal carcinoma (IDC) (A), invasive lobular carcinoma (ILC) (B), pleomorphic lobular carcinoma (PLC) (C, arrows), and mucinous carcinoma (MC) (D). The tumor cells of IDC and MC are cohesive and form clusters, whereas those of ILC and PLC are relatively dispersed with less cohesiveness. The entire cytoplasm of SRCs of IDC (case 1) stained positive for periodic acid-Schiff (E), and the intracytoplasmic lumen (ICL) of SRCs in ILC (case 5) specifically stained positive for alcian blue (F). Hematoxylin and eosin staining $(\mathrm{A}-\mathrm{D})$. Original magnification $\times 600(\mathrm{~A}-\mathrm{F})$.
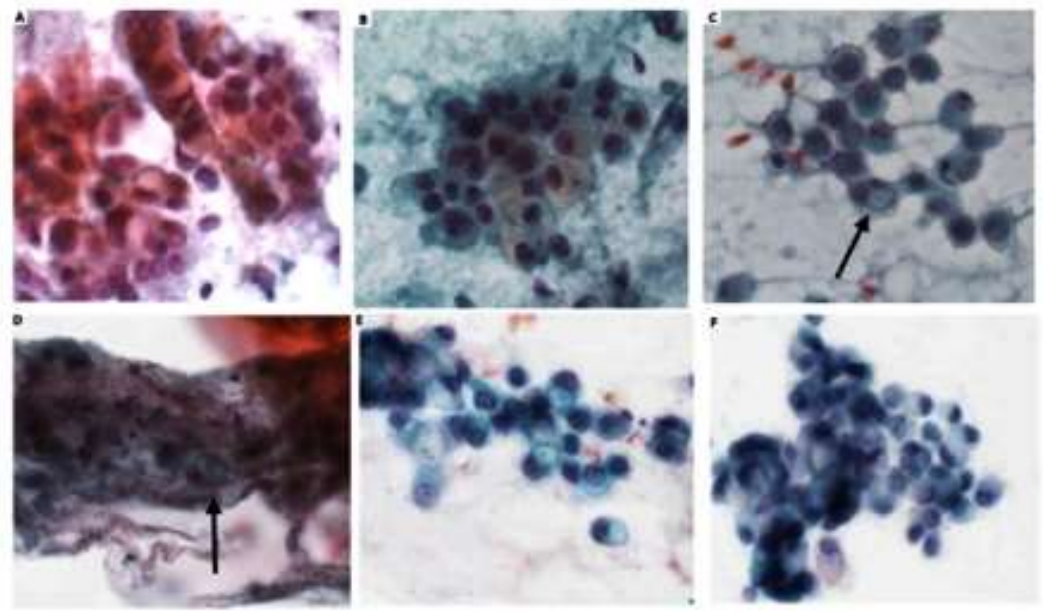

Fig. 2: Cytomorphology of signet ring cells (SRCs) in invasive ductal carcinoma (A, B), invasive lobular carcinoma (C, arrow), pleomorphic lobular carcinoma (D, arrow), and mucinous carcinoma (E, F) identified in fine needle aspiration biopsy smears. SRCs were categorized into intracytoplasmic lumen (ICL) type (A, C-E) and non-ICL type (B and F). ICL type SRCs are characterized by a "target" appearance because of the large ICL. Non-ICL type SRCs have an acidic muco-substance that diffusely fills the entire cytoplasm and displaces the nucleus to one edge of the cell, as seen in gastric carcinoma. Papanicolaou staining (A-F). Original magnification $\times 600(\mathrm{~A}-\mathrm{F})$. 